\title{
Development of new small-angle neutron scattering geometry with ring-shaped collimated beam for compact neutron source
}

\author{
Fumiaki Funama ${ }^{1, *}$, Yuya Adachi ${ }^{1}$, Seiji Tasaki $^{1}$, and Yutaka Abe ${ }^{1}$ \\ ${ }^{1}$ Department of Nuclear Engineering, Kyoto University, Nishikyo-ku, Katsura, Kyoto 615-8540, Japan
}

\begin{abstract}
Small-angle neutron scattering (SANS) is an important tool to investigate material properties in nanometer to micrometer scale. The opportunity to conduct SANS experiments is, however, limited because of the low number of available SANS beam lines. Compact neutron sources are expected to play a significant role to increase neutron scattering facilities including SANS beam lines. The problem is that the flux of compact neutron sources can be very low, which makes it difficult to measure scattered neutrons from a sample. A SANS geometry with ring-shaped collimated beam (r-SANS) is developed to conduct SANS experiments at very low flux neutron sources. By using ring-shaped collimated neutrons to hit a large sample, the scattered neutron flux becomes high on the ring center line because the scattered neutrons with each scattered angle overlaps on each point of the ring center line. By setting a ${ }^{3} \mathrm{He}$ point detector on the center line and shielding the surrounding of the small detection area well, high signal to noise ratio experiments are possible. In this paper, we show the concept of this new geometry and a preliminary experimental result of a glassy carbon sample taken with the r-SANS geometry constructed at Kyoto University proton Accelerator Neutron Source (KUANS).
\end{abstract}

\section{Introduction}

Small-angle neutron scattering (SANS) aims to study the structure of matter ranging from nanometer to micrometer scales [1]. The mesoscale structure is important to understand the macroscopic behaviour of matter. This technique offers several advantages since neutrons can penetrate into the samples deeply, are sensitive to isotopes and possess magnetic moments. Thus, using SANS and small angle x-ray scattering (SAXS) techniques complementarily, better understanding of the structure of matter in mesoscale is possible.

SANS experiments are normally done in large facilities with neutron sources. The number of SANS beamlines is very limited since nuclear spallation, reaction or fission phenomena are necessary for neutron production, which makes the facilities expensive. In the hope of cutting the cost, shrinking the size of the facilities and making SANS experiments available in smaller scale laboratories, compact neutron sources are being developed. There are several advantages in constructing SANS instruments at compact neutron sources. SANS experiments do not require high spatial and energy resolutions relatively. Because of that, utilizing more scattered neutrons is possible by summing up more neutrons in the same momentum transfer bin. The neutron emission area is much larger than its SAXS counterpart, which means that bigger samples can be measured. The target-station is also relatively easier to access and any shape of slit can be put just in front of the moderator. The residual radioactivity does not remain so much after the

\footnotetext{
* Corresponding author: funama.fumiaki.26e@kyoto-u.jp
}

experiment especially when a few $\mathrm{MeV}$ proton beam is used for the neutron production. By placing the slit near the moderator, environmental noise can be reduced since unnecessary neutrons can be stopped by the slit at the most up-stream part of the instrument. Moreover, the total length of the instrument can be greatly reduced, which enables us to utilize more neutrons during the experiments.

Although SANS with compact neutron sources offer a great potential, several challenges are needed to be addressed. The low neutron intensity of compact neutron sources is a problem for conducting SANS experiments. Several methods and devices were developed to increase the neutron intensity at the sample position. Multi-pinhole SANS geometries use a slit with several pinholes to collimate large area of neutrons and increase the incident neutron beams [2]. Focusing SANS geometries enable us to utilize more neutrons from a pinhole slit using ellipsoidal focusing mirrors [3]. These methods have its own advantages and disadvantages, which depend on different conditions such as the momentum transfer resolution needed in the experiment, the available neutron energy, and the scattering intensity of a sample. However, unlike x-ray production, in order to obtain thermal or cold neutrons, the moderation process is necessary which makes the neutron emission area much larger than x-ray in nature. Moreover, neutrons emitted from the moderator surface have brightness distribution but has no directional dependencies in effect. These intrinsic characteristics can be utilized more to compensate for the lack of neutron flux of compact neutron sources. In this paper, a new geometry for small-angle neutron 
scattering with ring-shaped collimated beams is proposed. The geometry and preliminary experimental results will be discussed.

\section{SANS geometry with ring-shaped collimated beam ( $r$-SANS geometry)}

A SANS geometry with ring-shaped collimated beams (rSANS) is developed in order to utilize more neutrons at compact neutron sources whose flux is very low. The schematic diagram of the geometry is shown in Fig. 1. The geometry is composed of a moderator, three ring-shaped slits, a sample and ${ }^{3} \mathrm{He}$ point detector placed next to each other. The distances between the moderator and the first slit, the first slit and the second slit, the second slit and the third slit, the third slit and the sample, and the sample and the detector are defined by $L M 1, L 12, L 23, L 3 s$ and $L s d$, respectively. The total instrument length, between the moderator surface and the detector, is defined by Lmd. The ${ }^{3} \mathrm{He}$ point detector is covered with a detector shielding material, which is made of $\mathrm{B}_{4} \mathrm{C}$. The shielding window is a hole through which scattered neutrons are able to be detected. The window has radius $r d$. The width of the three ring-shaped slits is $R w$. The center of all the components are aligned on the ring center line. The nominal scattering angle $\theta$ is then determined using $L 3 s$, $L s d$ and the nominal incident angle, which is determined by the first and third slit ring radii ( $R 1$ and $R 2), L 12$ and L23. The wavelength, $\lambda$, of the scattered neutron is calculated using time of flight method (TOF). These values are used to calculate the momentum transfer of scattered neutrons, $Q$, using equation (1).

$$
Q=\frac{4 \pi \sin \theta}{\lambda}
$$

By changing the detector position, $L s d$, we can change the measurable $Q$ range of the scattered neutrons in the measurement. The slits are made of neutron absorber material so that the neutron beam can only pass through the slits. The second slit is included to block neutrons that passes diagonally from the first to the third slit to reduce the smearing effect.

The mechanism for achieving high signal-to-noise $(\mathrm{S} / \mathrm{N})$ ratio experiments is explained as follows. The neutron beam only passes through the three rings in the tangent direction which provides a noiseless space on the ring center line. We put a zero-dimensional ${ }^{3} \mathrm{He}$ point detector, which has a higher counting ratio for neutrons and is less sensitive to gamma rays compared to a typical two-dimensional scintillation detector, on the line. Since the detection area of the detector, which is within the shielding window, is small, it is possible to minimize the noise from the environment by providing a good shielding around the detector. By measuring superimposed scattered beam on the beam center line, high $\mathrm{S} / \mathrm{N}$ ratio experiments are possible. Since the distances of the components from each other are flexible, the total length of the instrument can also be shortened in order to obtain more scattered neutron signals. This geometry can easily be adopted in other compact neutron sources with minimal
Despite the great possibilities that this geometry offers, there are also several limitations. Since the incident neutron beam hits the sample with the designed ringshape area, a large amount of sample is needed to cover the whole ring area. Considering the usual size of a moderator for a compact neutron source, a square sample size in the range of $5-15 \mathrm{~cm}$ is necessary. This may be difficult to achieve for biological samples. However, for other fields such as steel industries, which are interested on the average structure of their products, the sample size can be acceptable. Another limitation of the geometry is that anisotropic scattering of the sample is not distinguished because the detected neutrons are coming from different positions of the sample to the ring center line. Moreover, the momentum transfer resolution can be low depending on the size of the rings $(R 1, R 2$ and $R w)$, $r d$ and $L s d$. This low resolution may not be significant since achieving more scattered neutron intensity from a sample with a good $\mathrm{S} / \mathrm{N}$ ratio condition is usually the priority for SANS measurement using very low flux neutron sources. Lastly, the utilized solid angle for this rSANS geometry is usually small compared with a typical SANS instrument. In principle, however, it is possible to cover a larger range of scattered angle by putting or moving the ${ }^{3} \mathrm{He}$ point detector along the center axis. Since the scatterers are aligned in ring-shape, the observable scattering intensity of unit solid angle is the same as one of the pinhole geometry if the incident beam divergences for both cases are the same. Since r-SANS geometry uses cross-talk neutrons collimated with the three ring-shaped slits, it causes additional scattered neutrons from the sample.

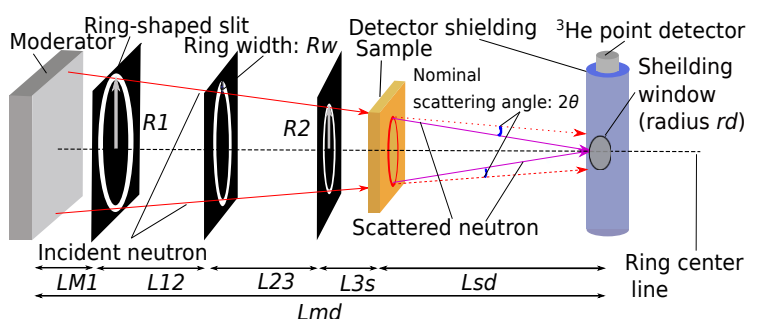

Fig. 1. Schematic diagram of r-SANS geometry

\section{Experiment}

In order to conduct the test measurements, we built an rSANS geometry at Kyoto University proton Accelerator Neutron Source (KUANS). KUANS is a compact neutron source with commercially available proton linac (AccSys Technology, Inc.) [4]. The neutron flux of KUANS is one of the lowest among available compact neutron sources for beam experiments [5]. With the same accelerator conditions of r-SANS measurement, the neutron intensity without any collimations was measured with ${ }^{3} \mathrm{He}$ point detector (6 atm, $1 \mathrm{inch}$ ) on site. The intensity was just a few hundred $\mathrm{n} / \mathrm{cm}^{2} / \mathrm{s}$ at $2.5 \mathrm{~m}$ away from the polyethylene moderator surface.

The geometry parameters for the experiment are shown in Table 1. The first slit diameter was decided by considering the neutron brightness of the moderator. The third collimator radius is decided by the sample size $(5 \mathrm{x}$ 
$5 \mathrm{~cm}^{2}$ ). The distance between the moderator surface and detector, $L m d$, was $2650 \mathrm{~mm}$, which is a lot shorter than normal SANS set-up, to utilize more neutrons. The distance between the sample and detector, $L s d$, is decided by the momentum transfer range of interest for our measurement. All ring-shaped slit widths were $4 \mathrm{~mm}$ in this experiment. As a sample, a glassy carbon plate (Alpha Aesar, product code: 038021, $1 \mathrm{~mm}$ (0.04in) thick, type 2) was chosen, which is widely used standard sample for SANS and SAXS measurement [6]. The thickness was 2 $\mathrm{mm}$ by piling the two plates up.

Table 1. Parameters in Fig.1 for r-SANS experiment

\begin{tabular}{|c|c|}
\hline Parameter & Value \\
\hline$R I$ & $42 \mathrm{~mm}$ \\
\hline$R 2$ & $21 \mathrm{~mm}$ \\
\hline$R w$ & $4 \mathrm{~mm}$ \\
\hline$L M 1$ & $45 \mathrm{~mm}$ \\
\hline$L 12$ & $1010 \mathrm{~mm}$ \\
\hline$L 23$ & $895 \mathrm{~mm}$ \\
\hline$L 3 s$ & $30 \mathrm{~mm}$ \\
\hline$L s d$ & $640 \mathrm{~mm}$ \\
\hline$L m d$ & $2620 \mathrm{~mm}$ \\
\hline$r d$ & $4.5 \mathrm{~mm}$ \\
\hline $2 \theta$ & $0.021 \mathrm{rad}$ \\
\hline
\end{tabular}

Figure 2 shows the TOF spectra of the direct beam, the scattered neutron and the background measurements. The direct beam of KUANS has a thermal peak at $1.14 \AA$. The scattered neutron from the glassy carbon sample has a stronger intensity than the background measurement. The background level is $1.58 \times 10^{-3}$ counts/s (5.68 counts/hour) for neutrons with wavelengths longer than $0.8 \AA$, which is very low because of the benefits of the $r$ SANS geometry. With the nominal scattering angle $(2 \theta)$ and the neutron transmission ratio of the glassy carbon sample, we can obtain the scattering intensity by using the following equation

$$
I(Q) \propto \frac{\frac{I_{s}(\lambda, 2 \theta)}{T(\lambda)}-I_{b}(\lambda, 2 \theta)}{I_{d}(\lambda)}
$$

where $I(Q)$ is the scattering intensity of a sample which is proportional to the differential cross section. $I_{s}(\lambda, 2 \theta)$

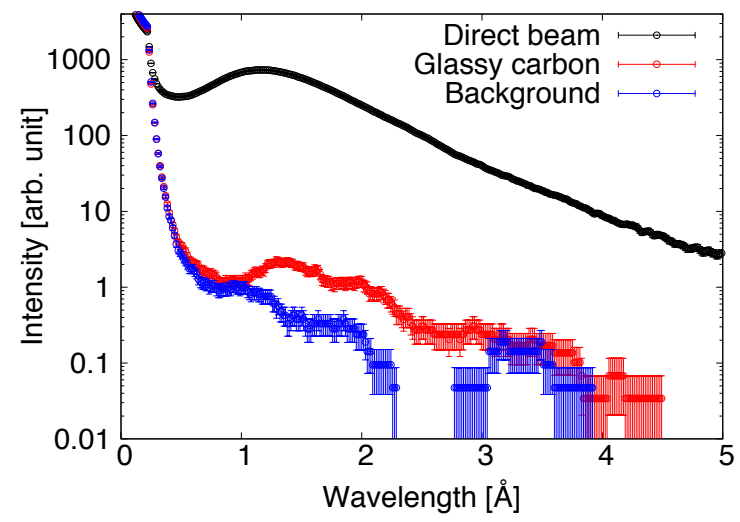

Fig. 2. TOF spectra of the direct beam, the scattered neutron from glassy carbon and the background measurements and $I_{b}(\lambda, 2 \theta)$ are the scattering intensity of the sample and the background measurements, respectively. $T(\lambda)$ and $I_{d}(\lambda)$ are the transmission ratio of the sample and the direct beam spectrum.

The measured scattering intensity of the glassy carbon sample is shown in Fig. 3 as well as the reference result of a SANS measurement for the same product sample [6]. The r-SANS experimental result is consistent with the published result although a discrepancy occurs when $Q$ becomes larger than $0.1[1 / \AA]$. This can be due to the effect of the oblique incident neutrons which pass through the three ring slits but do not have the same scattering angle values with that of the nominal scattering angle. These cross-talk neutron beams can enhance, however at the same time, smear the observed scattering intensity. The development of the analysis method is a subject for future research.

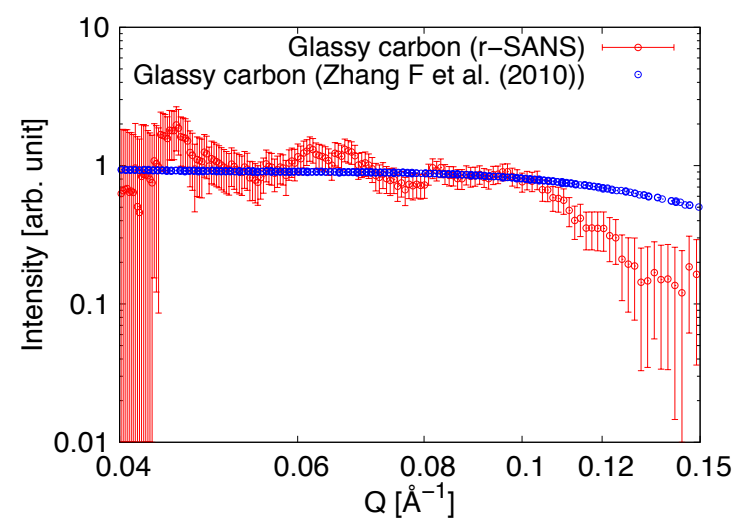

Fig. 3. Scattering intensity of a glassy carbon sample

\section{Conclusion}

In order to conduct SANS experiments at very low flux neutron sources, we propose a new geometry with ringshaped collimated beam (r-SANS). This geometry enables us to observe converging scattered neutrons from the sample at the noiseless ring center line with a zerodimensional ${ }^{3} \mathrm{He}$ point detector. As a test measurement, we constructed an r-SANS geometry at Kyoto University proton Accelerator Neutron Source (KUANS) which has one of the lowest neutron flux among available compact neutron sources for beam experiments. A glassy carbon sample was used for the measurement. Scattered neutrons from the sample was successfully observed and the result was consistent with the published result. However, the result is not statistically enough and does not have a good agreement with available experimental data in the large $Q$ region, $Q>0.1[1 / \AA]$. This disagreement can be due to the effect of the oblique incident neutrons which do not have the same scattering angle with the nominal scattering angle. The estimation of the smearing effect is a subject for the future research in order to improve this new geometry.

The authors are grateful to Mr. Hirose for the operation of KUANS. This work was partially supported by the Seamless Technology Transfer Program through Target driven R \& D, Japan Science and Technology Agency (JST). 


\section{References}

1. R. J. Roe. Methods of X-ray and neutron scattering in polymer science. 739. New York: Oxford University Press, (2000)

2. C. J. Glinka, J. M. Rowe, J. G. LaRock, J. Appl. Cryst. 19, 427 (1986)

3. B. Khaykovich, M. V. Gubarev, Y. Bagdasarova, B. D. Ramsey, D. E. Moncton, Nucl. Instrum. Methods Phys. Res., Sect. A, 631, 98 (2011)

4. S. Tasaki, T. Nagae, M. Hirose, Y. Yamashita, K. Hironaka, Y. Abe, Y. yamagata, Y. Otake, K. Hirota, Physics Procedia 60, 181 (2014)

5. I. S. Anderson, C. Andreani, J. M. Carpenter, G. Festa, G. Gorini, C. -K. Loong, R. Senesi, Physics Reports 654, 1 (2016)

6. F. Zhang, J. Ilavsky, G. G. Long, J. P. G. Quintana, A. J. Allen, P. R. Jemian, Metall. Mater. Trans. A 41, 1151 (2010) 